rev Psi

Revista de Psicología (UNLP)

https://revistas.unlp.edu.ar/revpsi

\title{
Reír para recordar: mejora de la memoria en relación con el humor
}

\author{
Anne-Lise Saive ${ }^{1}$ \\ Correspondencia \\ anne-lise.saive@umontreal.ca \\ Filiaciones institucionales \\ ${ }^{1}$ Université de Montreal (Canadá) \\ Traducción \\ Marina Trakas
}

\section{Resumen}

Las emociones positivas son valoradas y buscadas en nuestra vida diaria, pero aún no se comprende bien el rol funcional que juegan en la cognición humana. A pesar de ciertas similitudes, las emociones positivas y negativas parecen depender de vías neuronales distintas y, por tanto, podrían influir de manera diferente en funciones cognitivas como la memoria. En este artículo de revisión, se presentan los efectos cognitivos y los fundamentos neuronales de las emociones positivas, centrándose en las especificidades de las emociones inducidas por el humor. Posteriormente, se describe la influencia beneficiosa del humor sobre la memoria y se analizan los posibles mecanismos neuronales a través de los cuales el humor puede mejorar la memoria en el cerebro humano.

\section{Palabras clave}

risa | humor | cerebro humano | memoria | emociones positivas | redes cerebrales

\section{Cómo citar}

Saive, A.-L. (2021). Reír para recordar: mejora de la memoria en relación con el humor. Revista de Psicología, 20(1), 178192. HTTPS://DX.DOI.ORG/10.24215/ 2422572 XE125

\begin{tabular}{|c|c|}
\hline \multicolumn{2}{|c|}{ Proceso editorial } \\
\hline Recibido & 1ra decisión \\
\hline 18 may. 2020 & 6 jul. 2020 \\
\hline Aceptado & Publicado \\
\hline 1 may. 2021 & 31 jul. 2021 \\
\hline
\end{tabular}

Coordinadora del dossier

Marina Trakas (Instituto de Investigaciones Filosóficas, IIF-SADAF, Argentina)
ISSN

2422-572X

Licencia

Licencia de Cultura Libre CC-BY 4.0

(Compartir - Adaptar - Atribuir)

Entidad editora

RevPsi es una publicación de la

Facultad de Psicología (Universidad

Nacional de La Plata, Argentina) 


\section{Rir para lembrar: melhoria da memória relacionada ao humor}

\section{Resumo}

As emoções positivas são valorizadas e procuradas em nossa vida diária, mas seu papel funcional na cognição ainda é pouco compreendido. Apesar de certas semelhanças, as emoções positivas e negativas dependem de vias neuronais distintas e podem, portanto, influenciar de forma diferente as funções cognitivas, como a memória. Neste artigo de revisão, apresento primeiro os efeitos cognitivos e as bases neurais das emoções positivas, focalizando as especificidades das emoções induzidas por humor. Posteriormente, descrevo a influência benéfica do humor na memória e discuto os possíveis mecanismos neurais que podem melhorar a memória relacionada ao humor no cérebro humano.

\section{Palavras-chave}

riso | humor | cérebro humano | memória | emoções positivas | redes cerebrais

\section{Laughing to remember: humor-related memory improvement}

\section{Abstract}

Positive emotions are valued and sought after in our daily lives, but their functional role in cognition is still poorly understood. Despite common ground, positive and negative emotions would rely on distinct neuronal pathways and might therefore differently influence cognitive functions such as memory. In this review, I first present the cognitive effects and neural underpinnings of positive emotions, focusing on the specificities of humor-induced emotions. Second, I describe the beneficial influence of humor on memory, and I discuss potential neural mechanisms that can support humor-related memory improvement in the human brain.

\section{Keywords}

laughter | humour | human brain | memory | positive emotions | brain networks 


\section{Aspectos destacados del trabajo}

- Debido a sus distintas bases neuronales, las emociones positivas podrían actuar de manera diferente a las emociones negativas.

- La estimulación del humor da como resultado un estado emocional positivo que es beneficioso para el aprendizaje y el rendimiento de la memoria a largo plazo.

- El humor implica una red neuronal a gran escala que es no obstante específica, y que incluye áreas cerebrales vinculadas a las emociones, la memoria y la recompensa.

La memoria refiere a nuestra capacidad de codificar, almacenar y recuperar información de manera flexible. Sin memoria es imposible comunicarse, crear relaciones duraderas o mantener nuestra identidad. Los recuerdos no están fijados en nuestro cerebro, sino que se actualizan constantemente para adaptar y orientar nuestro comportamiento futuro. Las emociones y la cognición están estrechamente entrelazadas, ya que los procesos cognitivos son necesarios para provocar emociones y, simultáneamente, las emociones modulan la manera en que percibimos, recordamos y reaccionamos al entorno (Phelps, 2004; Phelps et al., 2006).

En el libro La expresión de las emociones en el hombre y los animales, Darwin definió las emociones como entidades discretas que nos permiten interactuar con flexibilidad y adaptarnos al mundo que nos rodea (Darwin, 1872). Actualmente, las emociones suelen concebirse como un estado interno desencadenado por un estímulo que induce respuestas somáticas y fisiológicas (Anderson y Adolphs, 2014; Barrett, 2014; Solomon, 2003). En psicología cognitiva, no obstante, no se ha profundizado en el estudio de las emociones positivas asociadas con un sentimiento subjetivo agradable, como la alegría, la felicidad, la satisfacción y el interés. Sin embargo, las emociones positivas contribuyen en gran medida a la calidad de vida de las personas (Diener y Larsen, 1993; Myers y Diener, 1995), mejoran el rendimiento cognitivo (Bradley et al., 1992; Cahill y McGaugh, 1995; Lang et al., 1995), y se han relacionado con rutas neuronales específicas en el cerebro humano (Anderson et al., 2003; Lewis et al., 2007; Small et al., 2003; Viinikainen et al., 2010). Por ello mismo, es fundamental ampliar nuestro conocimiento sobre las emociones positivas y revelar los mecanismos neuronales a partir de los cuales las emociones positivas modulan procesos cognitivos como la memoria.

Este artículo de revisión tiene como objetivo remediar dicha falta de análisis. Por un lado, se presentan los fundamentos neuronales de las emociones positivas y las especificidades de la risa y las emociones positivas relacionadas con el humor. Por el otro, se discuten los posibles mecanismos neuronales que subyacen la fuerte influencia del humor sobre la memoria a la luz de recientes estudios tanto comportamentales como funcionales realizados en humanos. 


\section{Representaciones neuronales superpuestas pero únicas de emociones positivas}

Las emociones se han conceptualizado a lo largo de dos dimensiones relacionadas: la valencia, que se extiende desde lo desagradable a lo agradable; y la excitación (arousal), que refiere al nivel de intensidad asociado con una emoción (Yik et al., 1999). De manera general, el procesamiento emocional recluta una gran red neuronal común que incluye la amígdala, el hipocampo y el parahipocampo, así como las cortezas prefrontal, temporal, cingulada anterior y el precuneus (George et al., 1995; Habel et al., 2005; Lane et al., 1997; Sang Hee Kim y Hamann, 2007). Sin embargo, la excitación y el placer han demostrado efectos neuronales específicos. Ciertos estudios funcionales han demostrado una actividad cerebral lateralizada relacionada con la valencia, con emociones positivas que se procesan predominantemente en el hemisferio izquierdo y emociones negativas en el hemisferio derecho (Wager et al., 2003). Otros estudios no han logrado demostrar una lateralización de la actividad neuronal relacionada con la valencia, sino que han revelado distintas vías neuronales asociadas con la valencia y con la excitación (Anderson et al., 2003; Lewis et al., 2007; Small et al., 2003; Viinikainen et al., 2010). Correlaciones en forma de U entre las escalas de valoración de valencia y la señal BOLD se han reportado de manera bilateral en la corteza prefrontal, la corteza cingulada anterior, la corteza orbitofrontal y la ínsula. La excitación se ha relacionado con la actividad neuronal en la amígdala cuando los estímulos se califican como agradables. Además, se ha encontrado que los estados afectivos positivos reclutan el circuito dopaminérgico, el cual está involucrado en múltiples formas de estados emocionales positivos y bienestar subjetivo (Depue y Collins, 1999).

Estudios recientes van más allá de las teorías que postulan que emociones específicas emergen a partir de la codificación neuronal de la valencia (Barrett y Russell, 1999; Russell, 1980), pues han identificado firmas neuronales únicas de emociones básicas discretas como el miedo, la felicidad, la tristeza o la sorpresa. Estos estudios de neuroimagen han aplicado algoritmos de aprendizaje automático para revelar redes cerebrales a gran escala espacialmente separables, particularmente en regiones cerebrales frontales, que pueden predecir y, por lo tanto, distinguir emociones discretas con altos niveles de precisión (Kassam et al., 2013; Kragel y LaBar, 2015, 2016; Saarimäki et al., 2016). Estos resultados refuerzan modelos categóricos en los que las emociones se caracterizan por distintos sustratos neuronales y fisiológicos (Ekman, 1992). Dentro de las emociones positivas, la especificidad de los correlatos neuronales de la felicidad ha sido la más estudiada (George et al., 1995; Habel et al., 2005; Kim y Hamann, 2007; Lane et al., 1997). Se ha encontrado que los patrones únicos de activaciones en las cortezas prefrontal, cingulada y temporal, los cuales no están presentes en las emociones negativas como el disgusto y la tristeza, constituyen la base del sentimiento subjetivo de felicidad. En resumen, aunque las emociones comparten algunos sustratos neuronales comunes, parecen también depender de patrones neuronales específicos a cada emoción que son lo suficientemente 
diferentes como para permitir que un algoritmo de aprendizaje automático los distinga de manera significativa.

\section{¿Por qué estudiar la risa y el humor?}

La interacción entre las emociones positivas y la memoria no ha sido estudiada en profundidad en parte porque es extremadamente difícil provocar emociones positivas, como la alegría y la satisfacción, en un entorno artificial y controlado como lo es el laboratorio. Esto no solo necesita de la creación de un vasto conjunto de datos bien caracterizados que provoque emociones positivas de manera fiable, sino que también requiere la capacidad de detectar y cuantificar estados emocionales. Entre las emociones positivas, la risa puede verse como la única expresión omnipresente de alegría, observable en todas las culturas, especies y generaciones que surge en los seres humanos alrededor del cuarto mes de vida (Ruch y Ekman, 2001). Además, la risa promueve fuertemente la vinculación y facilita las interacciones sociales (Martin y Lefcourt, 1983, 2004; Scott et al., 2014). En los seres humanos, la risa también se puede utilizar como un mecanismo de afrontamiento (coping mechanism), para aliviar el estrés y anular momentáneamente las emociones negativas. Según la hipótesis de la reversión (undoing hypothesis), reír en situaciones estresantes ayuda a regular negativamente las emociones negativas persistentes y atenúa los síntomas relacionados con el estrés a largo plazo (Fredrickson et al., 2000; ZanderSchellenberg et al., 2020).

La risa puede entonces constituir un marcador fiable ideal de un estado emocional positivo. Aunque la risa puede ser provocada por cosquillas o por el gas de la risa, la mayoría de los estudios se han centrado en la risa provocada por el humor, un proceso emocional complejo de orden superior (Vrticka et al., 2013; Wild et al., 2003). El humor es en general considerado como una interacción social que comprende facetas cognitivas (detección de incongruencias), emocionales (alegría) y expresivas (risa) (Martin, 2007). Estudios recientes han revelado que es un recurso beneficioso para la salud, ya que puede ayudar a prevenir el desarrollo de enfermedades e infecciones cardiovasculares (Romundstad et al., 2016) y permite afrontar mejor eventos estresantes (Martin y Lefcourt, 1983, 2004). Además, la risa inducida por el humor también está relacionada con mejoras significativas y duraderas en el estado de ánimo depresivo, la ansiedad, el alivio del dolor, el aumento de la felicidad y la mejora de la autoestima (SH Kim et al., 2015; Ko y Youn, 2011; Tse et al., 2010).

El humor y la risa podrían constituir herramientas fiables y potentes para utilizar emociones positivas en la estimulación de la formación de los recuerdos, y para crear, de esta manera, experiencias de aprendizaje más eficaces. Este tipo de investigación es de suma importancia para la obtención de nuevos conocimientos fundamentales sobre el funcionamiento de la memoria, pues podría ayudar a optimizar las estrategias de aprendizaje y las terapias que combaten la pérdida de memoria tanto en poblaciones sanas como clínicas. 


\section{El humor implica áreas cerebrales vinculadas a las emociones, la memoria y la recompensa}

El hecho de que personas de diferentes culturas perciban y utilicen el humor de manera distinta en la vida cotidiana constituye un desafío para estudiar el humor científicamente (Martin, 2007; Yue et al., 2016). Martin y colegas han definido cuatro tipos de humor: humor afiliativo, que refleja la tendencia a bromear y divertir a los demás; humor auto-estimulante, que representa el uso del humor como regulador emocional; humor agresivo, que refleja la inclinación a criticar, burlarse y manipular a los demás con humor; y el humor autodestructivo, que se relaciona con la predisposición a hacer bromas para devaluarse. Las diferencias individuales en el uso de estas distintas formas de humor se han relacionado con efectos positivos o negativos en la salud y el bienestar de las personas (Martin et al., 2003). Las personas que favorecen el humor auto-estimulante demostraron mejores mecanismos de afrontamiento frente al estrés, y se caracterizan por poseer personalidades alegres que se toman las cosas a la ligera. Se ha descubierto también que el uso del humor afiliativo reduce eficazmente las tensiones sociales. Por el contrario, los estilos de humor tanto agresivos como autodestructivos se han identificado como perjudiciales para el bienestar de las personas.

Además de la caracterización de los tipos de humor que la gente tiende a preferir y de sus respectivas implicaciones para la salud, se han realizado algunos intentos para conceptualizar los procesos fundamentales que subyacen al humor en su conjunto (Suls, 1972; Wyer y Collins, 1992). Estos autores postulan que la percepción de que algo es divertido (es decir, la estimulación del humor) se basa en un proceso de dos etapas que comprende por un lado la comprensión y por el otro la elaboración del humor. La comprensión del humor se refiere a (a) la detección de incongruencias entre la información esperada según las particularidades del contexto y la información efectivamente recibida; y (b) la resolución de estas incongruencias a partir de reinterpretaciones basadas en conceptos y conocimientos de otros dominios de especialización. Posteriormente tiene lugar la segunda etapa, es decir, la elaboración del humor. En esta fase se generan y desarrollan las implicaciones de esta reinterpretación, lo cual provoca el sentimiento de diversión.

La gran mayoría de los estudios de neuroimagen que investigan el humor han utilizado todo tipo de material humorístico, incluidos chistes, bromas y dibujos animados, sin distinguir su base motivacional o propósito. Dichos estudios han encontrado que distintas áreas del cerebro están asociadas con la comprensión y con la elaboración del humor (Bartolo et al., 2006; Bekinschtein et al., 2011; Chan et al., 2012, 2013; Franklin y Adams, 2011; Goel y Dolan, 2001; Mobbs et al., 2003; Watson et al., 2007). La comprensión de un chiste, en tanto que tarea cognitiva, recluta los giros frontales inferiores bilaterales y el giro frontal superior izquierdo, así como la unión temporoparieto-occipital. La sensación de diversión que ocurre durante la elaboración del humor involucra la corteza prefrontal ventromedial izquierda, la sustancia negra, la ínsula bilateral, la amígdala y las cortezas parahipocampales. Curiosamente, se 
encontró que el grado de intensidad del humor o nivel de diversión, se correlaciona positivamente con la actividad neuronal en las regiones del cerebro relacionadas con la recompensa, como el núcleo accumbens (Bekinschtein et al., 2011; Franklin y Adams, 2011; Mobbs et al., 2003). Estos resultados son consistentes con la idea de que cuanto mejor las personas comprenden y elaboran el chiste, lo disfrutan más y se sienten más divertidos y recompensados. En resumen, la estimulación del humor genera un estado emocional positivo y activa una red neuronal a gran escala que comprende regiones cerebrales asociadas a la memoria, la emoción y la recompensa.

\section{La risa sin humor no involucra ni la memoria ni la recompensa}

La percepción de la risa por sí sola no activa una red neuronal tan vasta y, lo que es más importante, no recluta al sistema dopaminérgico responsable de la recompensa y la motivación (Lavan et al., 2016; McGettigan et al., 2015; Wild et al., 2015; Wild et al., 2003), del cual se sabe que mejora el aprendizaje y la memoria (Gruber et al., 2014, 2016; Kang et al., 2009; Patil et al., 2017). La risa se sustenta en sistemas neuronales complejos, lo que permite la existencia de dos formas distintas de risa dependiendo de la manera en que se provoca: (1) involuntariamente, en respuesta a eventos externos, lo que se conoce como risa espontánea o real, y (2) voluntariamente, en situaciones sociales, con el objetivo deliberado de crear lazos afectivos, lo que ha sido denominado risa social (Gervais y Wilson, 2005; Scott et al., 2014). En los primeros estudios científicos de la risa, los neurólogos se centraron en sus variantes patológicas (Brissaud, 1895; Nothnagel, 1889; Poeck, 1969). La risa patológica es una forma de risa espontánea que surge en situaciones inapropiadas y en ausencia de control voluntario. Se ha asociado con lesiones cerebrales en el tronco encefálico, el tálamo y la corteza frontal. Los estudios de estimulación eléctrica cerebral invasiva han vinculado los ataques de risa con la corteza cingulada anterior y regiones cerebrales profundas, como la circunvolución fusiforme y el parahipocampo (Arroyo et al., 1993; Sem-Jacobsen, 1968).

La mayoría de los estudios más recientes que exploran la risa han utilizado como método la escucha pasiva de la risa espontánea, y han revelado que la percepción por sí sola induce un estado de alegría, que involucra áreas cerebrales emocionales como la amígdala y la ínsula (Sander et al., 2003; Sander y Scheich, 2005; Wattendorf et al., 2015). Estos estudios también han identificado una gran red neuronal que sustenta la escucha pasiva de la risa espontánea y que reúne las cortezas hipotálamo, mesencéfalo, motora, pre-motora y somatosensorial, así como regiones temporales superiores (Meyer et al., 2007; Warren et al., 2006; Wild et al., 2003). La actividad en las cortezas sensoriomotora y prefrontal medial se ha relacionado con la evaluación del significado de la risa (Lavan et al., 2016; McGettigan et al., 2015; Szameitat et al., 2011). Se cree que la participación sensoriomotora refleja la capacidad de los participantes para discriminar entre la risa espontánea y la risa social, mientras que la actividad de la corteza prefrontal medial parece disminuir linealmente en función de la autenticidad de la risa. Por el contrario, las cortezas auditivas se reclutan 
principalmente durante la escucha de la risa espontánea, y su participación aumenta linealmente con la autenticidad de la risa (Lavan et al., 2016; McGettigan et al., 2015). En conclusión, la risa auténtica y espontánea involucra una red neuronal restringida que no incluye las regiones cerebrales de memoria y de recompensa.

\section{La influencia beneficiosa del humor sobre la memoria}

De todas nuestras experiencias diarias, solo algunas de ellas se transformarán en recuerdos duraderos que podremos recordar más tarde. Los eventos emocionales gozan de un lugar privilegiado en nuestra memoria, ya que se recuerdan con mayor detalle y vivacidad que la mayoría de los eventos neutrales (Kensinger, 2009; Saive et al., 2014), lo cual refleja un procesamiento prioritario de eventos emocionales a costa de otra información neutral considerada menos importante en la situación presente. A menudo se considera que la excitación emocional es el principal factor responsable de este efecto, ya que se ha demostrado de manera contundente que los estímulos que despiertan dicha excitación son más persistentes que los que no la provocan (Bradley et al., 1992; Cahill y McGaugh, 1995; Lang et al., 1995). Por otro lado, la valencia de las emociones asociadas a los recuerdos también tiene un impacto en la memoria. Los estímulos negativos parecen recordarse mejor que los positivos cuando se controla la excitación (Christianson y Fällman, 1990; Kern et al., 2005). Sin embargo no hay un verdadero consenso sobre este hallazgo, ya que también se ha demostrado el efecto contrario (Bradley et al., 1992; Lang et al., 1995).

Aunque poco estudiado, el humor que conduce a emociones positivas se ha relacionado sólidamente con un mejor rendimiento de la memoria en poblaciones tanto sanas como clínicas. Este efecto beneficioso se ha demostrado mediante dibujos animados humorísticos (Schmidt, 2002; Schmidt y Williams, 2001; Takahashi y Inoue, 2009), videos (Bains et al., 2014, 2015) y oraciones (Schmidt, 1994). Bains y colegas $(2014,2015)$ concluyeron que la visualización de un video humorístico mejora la memoria a corto plazo (capacidad de aprendizaje, recuerdo diferido y reconocimiento visual) en poblaciones sanas, ancianas y diabéticas. Investigaciones complementarias han revelado un efecto a largo plazo significativo del humor sobre la memoria, al demostrar una mayor consolidación de la información humorística después de un período de 12 horas (Chambers y Payne, 2014). Por otro lado, los estudios de memoria que utilizan dibujos animados han mostrado que la detección y resolución de incongruencias, es decir, el proceso de apreciación del humor en dos pasos, no son suficientes para mejorar la memoria: "la percepción del humor está más estrechamente relacionada con el desempeño de la memoria que la resolución putativa de dibujos animados que, por definición, no contienen incongruencias (los dibujos animados literales) o contienen incongruencias que no se resuelven fácilmente (los dibujos animados raros)" (Schmidt y Williams, 2001, p. 310). La resolución de las incongruencias debe conducir a la diversión y al entretenimiento para que tenga un efecto en la memoria. Esto último es coherente con la idea de que el efecto del humor sobre la memoria está 
impulsado principalmente por la excitación que éste provoca. No obstante, aún se carece de evidencia directa para respaldar dicha afirmación.

Los efectos beneficiosos del humor también se han estudiado en el aula con alumnos de diferentes edades. Se ha demostrado que el humor ayuda a los estudiantes a mantenerse concentrados y comprometidos durante las clases y aumenta la participación y el aprendizaje (Bolkan et al., 2018; Goodboy et al., 2015). Sin embargo, hay muchos parámetros que deben tenerse en cuenta al utilizar el humor en el aula. Para garantizar un efecto positivo en el aprendizaje y el rendimiento, los estudiantes deben reconocer y resolver los mensajes humorísticos. El humor provocará así respuestas emocionales positivas y aumentará la motivación. Además, el contenido del mensaje es importante ya que tendrá un efecto beneficioso significativo solo cuando aborde el tema del curso.

\section{Mecanismos neuronales potenciales subyacentes a la mejora de la memoria relacionada con el humor}

El humor es un proceso emocional superior que estimula de manera contundente a la memoria. Aunque no existe aún una explicación mecanicista de este efecto, es posible plantear la siguiente hipótesis sobre las tres maneras en que el humor mejora la memoria durante la codificación, la consolidación y la recuperación de la misma.

En primer lugar, se ha descubierto que el humor disminuye los niveles de estrés al reducir la liberación de cortisol y aumentar la liberación de oxitocina (Riem et al., 2012; Sripada et al., 2013), lo cual está relacionado con un mejor rendimiento cognitivo y mnémico (Ford et al., 2012; Newcomer et al., 1999; Shields et al., 2017). Una concentración moderada de cortisol puede fortalecer la potenciación a largo plazo en el hipocampo y la amígdala, lo que ciertamente mejora la consolidación de la memoria (Joëls y Krugers, 2007). La disminución del nivel de cortisol puede también favorecer la recuperación de la memoria al aumentar la actividad del hipocampo y la corteza prefrontal (Oei et al., 2007). Además, los niveles elevados de oxitocina pueden desempeñar un papel en la mejora de la memoria al aumentar la conectividad funcional entre la amígdala y las regiones asociadas con la memoria, como el hipocampo y la corteza prefrontal medial (Riem et al., 2012; Sripada et al., 2013).

En segundo lugar, el humor provoca respuestas positivas y estimulantes que aumentan la prominencia y la relevancia emocional de cualquier material que es codificado a continuación. El humor captura la atención, lo que aumenta directamente la probabilidad de que la información se considere relevante y, por lo tanto, se almacene en la memoria (Pinheiro et al., 2017). La excitación puede aumentar la excitabilidad de la amígdala al inducir una respuesta noradrenérgica elevada, la cual induce al hipocampo y la corteza prefrontal a mejorar la codificación y el almacenamiento de los recuerdos (Kensinger, 2009; LaBar y Cabeza, 2006; Phelps, 2004; Strange y Dolan, 2004). Además, la codificación a largo plazo de los recuerdos emocionales también podría estar relacionada con una mayor 
consolidación durante el sueño, lo que aumenta directamente las posibilidades de que un evento sea recordado posteriormente. Los cambios en la conectividad entre la amígdala y el hipocampo favorecerían la reproducción (replay) emocional y amplificarían la transferencia de estos recuerdos desde el hipocampo hasta las regiones neocorticales (Holland y Lewis, 2007; Paré et al., 2002). El grado de actividad de la amígdala predice directamente el rendimiento de la memoria, y hallazgos recientes han indicado que el reclutamiento mejorado de la amígdala puede persistir en el tiempo y mejorar de manera prospectiva la forma en que se codifica y recuerda información nueva y neutral (Tambini et al., 2016). Nuevos estudios conductuales tienden a confirmar esta idea al mostrar que la combinación de material neutro y humorístico mejora la memoria para la información neutral (Carlson, 2011; Suzuki y Heath, 2014; Takahashi y Inoue, 2009). Además, la respuesta emocional positiva relacionada con el humor involucraría áreas frontales y parietales asociadas con la memoria episódica y el procesamiento autorreferencial, que no son reclutadas por las emociones negativas (Kensinger y Corkin, 2004; Mickley y Kensinger, 2008). Curiosamente, el aumento de la respuesta noradrenérgica en la amígdala inducido por el humor es necesario para que el cortisol tenga un efecto sobre la memoria. En un contexto neutral o no excitante, la disminución del cortisol no será suficiente para afectar a la memoria. El hecho de que el humor actúe sobre estas dos hormonas aumenta en gran medida la potencia para mejorar la consolidación de los recuerdos.

Por último, la diversión y el entretenimiento relacionados con el humor inducen recompensa y motivación al reclutar el sistema dopaminérgico. Hallazgos recientes han revelado que la motivación induce una mayor actividad del núcleo accumbens, una región del cerebro que libera dopamina y está asociada con el humor, y que también promueve la memoria (Gruber et al., 2014). La dopamina conduce a un sesgo de atención y codificación hacia la información relacionada con las recompensas y mejora la consolidación dependiente del hipocampo (Gruber et al., 2016; Lisman y Grace, 2005). Un estudio reciente ha demostrado que fuertes interacciones entre la amígdala y el núcleo accumbens se asocian con el procesamiento de información positiva versus negativa (Beyeler et al., 2016). Los estudios mencionados también han demostrado que la dopamina puede beneficiar la retención de recuerdos de eventos que no están directamente relacionados con la recompensa, pero que ocurren cercanos en el tiempo. Por tanto, es posible que, al inducir un contexto gratificante, el humor aumente la consolidación de la memoria así como también la retención de información en general.

En conclusión, el humor puede ser considerado como un fuerte inductor emocional capaz de provocar un estado emocional positivo y duradero, acompañado de una reducción del estrés y un aumento de los niveles de motivación y atención. Al activar la amígdala de manera sostenida y reclutar circuitos dopaminérgicos, el humor podría utilizarse antes del aprendizaje para reforzar la formación y consolidación de la memoria, lo cual ayudaría de esta manera a crear experiencias de aprendizaje más efectivas en medios tanto educativos como clínicos. 


\section{Financiación}

ALS cuenta con la financiación del Fonds de Recherche du Québec - Nature et Technologies, una Beca de Excelencia del IVADO y una Beca de Excelencia de UNIQUE. Las instituciones proveedoras de fondos no tuvieron ningún papel en el diseño del artículo, la decisión de publicarlo o la preparación del manuscrito.

\section{Agradecimientos}

Deseo dar las gracias a C. El Khantour por su valiosa ayuda en la búsqueda bibliográfica específica.

\section{Referencias}

Anderson, A. K., Christoff, K., Stappen, I., Panitz, D., Ghahremani, D. G., Glover, G., Gabrieli, J. D. E. y Sobel, N. (2003). Dissociated neural representations of intensity and valence in human olfaction. Nature Neuroscience, 6(2), 196-202. HTTPS://DOI.ORG/10.1038/NN1001

Arroyo, S., Lesser, R. P., Gordon, B., Uematsu, S., Hart, J., Schwerdt, P., Andreasson, K. y Fisher, R. S. (1993). Mirth, laughter and gelastic seizures. Brain, 116(4), 757-780.

Bains, G. S., Berk, L. S., Daher, N., Lohman, E., Schwab, E., Petrofsky, J. y Deshpande, P. (2014). The effect of humor on short-term memory in older adults: A new component for whole-person wellness. Advances in Mind-Body Medicine, 28(2), 16-24.

Bains, G. S., Berk, L. S., Lohman, E., Daher, N., Petrofsky, J., Schwab, E. y Deshpande, P. (2015). Humors effect on short-term memory in healthy and diabetic older adults. Alternative Therapies in Health and Medicine, 21(3), 16-25.

Barrett, L. F. y Russell, J. A. (1999). The structure of current affect: Controversies and emerging consensus. Current Directions in Psychological Science, 8(1), 10-14.

Bartolo, A., Benuzzi, F., Nocetti, L., Baraldi, P. y Nichelli, P. (2006). Humor comprehension and appreciation: An fMRI study. Journal of Cognitive Neuroscience, 18(11), 1789-1798. HTTPs:// DOI.ORG/10.1162/JOCN.2006.18.11.1789

Bekinschtein, T. A., Davis, M. H., Rodd, J. M. y Owen, A. M. (2011). Why clowns taste funny: The relationship between humor and semantic ambiguity. Journal of Neuroscience, 31(26), 9665-9671. HTTPS://DOI.ORG/ 10.1523/JNEUROSCI.5058-10.2011
Beyeler, A., Namburi, P., Glober, G. F., Simonnet, C., Calhoon, G. G., Conyers, G. F., Luck, R., Wildes, C. P. y Tye, K. M. (2016). Divergent routing of positive and negative information from the amygdala during memory retrieval. Neuron, 90(2), 348-361. HTTPS://DOI.ORG/10.1016/J.NEURON.2016.03.004

Bolkan, S., Griffin, D. J. y Goodboy, A. K. (2018). Humor in the classroom: The effects of integrated humor on student learning. Communication Education, 67(2), 144-164. HTTPS://DOI.ORG/ $10.1080 / 03634523.2017 .1413199$

Bradley, M. M., Greenwald, M. K., Petry, M. C. y Lang, P. J. (1992). Remembering pictures: Pleasure and arousal in memory. Journal of Experimental Psychology. Learning, Memory, and Cognition, 18(2), 379-390.

Brissaud, E. (1895). Leçons sur les maladies nerveuses. En Vingt-quatrième leçon, Tics et spasms cloniques de la face (p. 446-468).

Cahill, L. y McGaugh, J. L. (1995). A novel demonstration of enhanced memory associated with emotional arousal. Consciousness and Cognition, 4(4), 410-421. HTTPS://DOI.ORG/ 10.1006/CCOG.1995.1048

Carlson, K. (2011). The impact of humor on memory: Is the humor effect about humor? Psychology Faculty Publications, 21. HTTPS:// SCHOLAR.VALPO.EDU/PSYCH_FAC_PUB/21

Chambers, A. M. y Payne, J. D. (2014). Laugh yourself to sleep: Memory consolidation for humorous information. Experimental Brain Research, 232(5), 1415-1427. HTTPS://DOI.ORG/10.1007/s00221- 
Chan, Y.-C., Chou, T.-L., Chen, H.-C. y Liang, K.-C. (2012). Segregating the comprehension and elaboration processing of verbal jokes: An fMRI study. Neurolmage, 61(4), 899-906. HTTPs:// DOI.ORG/10.1016/J.NEUROIMAGE.2012.03.052

Chan, Y.-C., Chou, T.-L., Chen, H.-C., Yeh, Y.-C. Lavallee, J. P., Liang, K.-C. y Chang, K.-E. (2013). Towards a neural circuit model of verbal humor processing: An fMRI study of the neural substrates of incongruity detection and resolution. NeuroImage, 66, 169-176. HTTPS://DOI.ORG/ 10.1016/I.NEUROIMAGE.2012.10.019

Christianson, S.-Å. y Fällman, L. (1990). The role of age on reactivity and memory for emotional pictures. Scandinavian Journal of Psychology, 31(4), 291-301. HTTPS://DOI.ORG/10.1111/J.14679450.1990.Тво0841.X

Depue, R. A. y Collins, P. F. (1999). Neurobiology of the structure of personality: Dopamine, facilitation of incentive motivation, and extraversion. Behavioral and Brain Sciences, 22(3), 491-517; discussion 518-569. HTTPS://DOI.ORG/ $10.1017 /$ so140525X99002046

Diener, E. y Larsen, R. (1993). The experience of emotional well-being. En M. Lewis y J.M. Haviland (Eds.), Handbook of emotions (pp. 405-415). Guilford.

Ekman, P. (1992). An argument for basic emotions. Cognition \& Emotion, 6(3-4), 169-200.

Ford, T. E., Ford, B. L., Boxer, C. F. y Armstrong (2012). Effect of humor on state anxiety and math performance. Humor, 25(1), 59-74. HTTPS:// DOI.ORG/10.1515/HUMOR-2012-0004

Franklin, R. G. y Adams, R. B. (2011). The reward of a good joke: Neural correlates of viewing dynamic displays of stand-up comedy. Cognitive, Affective, \& Behavioral Neuroscience, 11(4), 508-515. HTTPS://DOI.ORG/10.3758/S13415-011-0049-7

Fredrickson, B. L., Mancuso, R. A., Branigan, C. y Tugade, M. M. (2000). The undoing effect of positive emotions. Motivation and Emotion, 24(4), 237-258. HTTPS://DOI.ORG/10.1023/ A:1010796329158

George, M. S., Ketter, T. A., Parekh, P. I., Horwitz, B. Herscovitch, P. y Post, R. M. (1995). Brain activity during transient sadness and happiness in healthy women. The American Journal of Psychiatry, 152(3), 341-351. HTTPS://DOI.ORG/10.1176/ AJP.152.3.341

Gervais, M. y Wilson, D. S. (2005). The evolution and functions of laughter and humor: A synthetic approach. The Quarterly Review of Biology, 80(4), 395-430. HTTPS://DOI.ORG/10.1086/498281
Goel, V. y Dolan, R. J. (2001). The functional anatomy of humor: Segregating cognitive and affective components. Nature Neuroscience, 4(3), 237-238. HTTPS://DOI.ORG/10.1038/85076

Goodboy, A. K., Booth-Butterfield, M., Bolkan, S. y Griffin, D. J. (2015). The role of instructor humor and students' educational orientations in student learning, extra effort, participation, and out-ofclass communication. Communication Quarterly, 63(1), 44-61. HTTPS://DOI.ORG/10.1080/ $\underline{01463373.2014 .965840}$

Gruber, M. J., Gelman, B. D. y Ranganath, C. (2014). States of curiosity modulate hippocampusdependent learning via the dopaminergic circuit. Neuron, 84(2), 486-496. HTTPs://DOI.ORG/ 10.1016/J.NEURON.2014.08.060

Gruber, M. J., Ritchey, M., Wang, S.-F., Doss, M. K. y Ranganath, C. (2016). Post-learning hippocampal dynamics promote preferential retention of rewarding events. Neuron, 89(5), 1110-1120. HTTPS://DOI.ORG/10.1016/J.NEURON.2016.01.017

Habel, U., Klein, M., Kellermann, T., Shah, N. J. y Schneider, F. (2005). Same or different? Neural correlates of happy and sad mood in healthy males. NeuroImage, 26(1), 206-214. HTTPs://DOI.ORG/ 10.1016/J.NEUROIMAGE.2005.01.014

Holland, P. y Lewis, P. A. (2007). Emotional memory: Selective enhancement by sleep. Current Biology, 17(5), R179-R181. HTTPS://DOI.ORG/10.1016/ L.CUB.2006.12.033

Joëls, M. y Krugers, H. J. (2007). LTP after stress: Up or down? Neural Plasticity, 2007, artículo 93202. HTTPS://DOI.ORG/10.1155/2007/93202

Kang, M. J., Hsu, M., Krajbich, I. M., Loewenstein, G., McClure, S. M., Wang, J. T. y Camerer, C. F. (2009). The wick in the candle of learning: Epistemic curiosity activates reward circuitry and enhances memory. Psychological Science, 20(8), 963-973. H T TPS:// D OI.ORG/10.1111/J.14679280.2009.02402.X

Kassam, K. S., Markey, A. R., Cherkassky, V. L., Loewenstein, G. y Just, M. A. (2013). Identifying emotions on the basis of neural activation. PLoS ONE, 8(6), e66032. HTTPS://DOI.ORG/10.1371/ IOURNAL.PONE.0066032

Kragel, P. A. y LaBar, K. S. (2015). Multivariate neural biomarkers of emotional states are categorically distinct. Social Cognitive and Affective Neuroscience, 10(11), 1437-1448. ㅍTㅇ:// DOI.ORG/10.1093/SCAN/NSVO32

Kragel, P. A. y LaBar, K. S. (2016). Decoding the nature of emotion in the brain. Trends in Cognitive Sciences, 20(6), 444-455. HTTPS://DOI.ORG/ $\underline{10.1016 / \text { I.TICS.2016.03.011 }}$ 
Kensinger, E. A. (2009). Remembering the details: Effects of emotion. Emotion Review, 1(2), 99-113. HTTPS://DOI.ORG/10.1177/1754073908100432

Kensinger, E. A. y Corkin, S. (2004). Two routes to emotional memory: Distinct neural processes for valence and arousal. Proceedings of the National Academy of Sciences of the United States of America, 101(9), 3310-3315. HTTPS://DOI.ORG/10.1073/ PNAS.0306408101

Kern, R. P., Libkuman, T. M., Otani, H. y Holmes, K. (2005). Emotional stimuli, divided attention, and memory. Emotion, 5(4), 408-417. HTTPs:// DOI.ORG/10.1037/1528-3542.5.4.408

Kim, S. H., Kim, Y. H. y Kim, H. J. (2015). Laughter and stress relief in cancer patients: A pilot study. Evidence-based complementary and alternative medicine. ECAM, 2015, artículo 864739. HTTPS:/ DOI.ORG/10.1155/2015/864739

Kim, Sang Hee y Hamann, S. (2007). Neural correlates of positive and negative emotion regulation. Journal of Cognitive Neuroscience, 19(5), 776-798. HTTPS://DOI.ORG/10.1162/JOCN.2007.19.5.776

Ko, H.-J. y Youn, C.-H. (2011). Effects of laughter therapy on depression, cognition and sleep among the community-dwelling elderly. Geriatrics of Gerontology International, 11(3), 267-274

LaBar, K. S. y Cabeza, R. (2006). Cognitive neuroscience of emotional memory. Nature Reviews. Neuroscience, 7(1), 54-64. HTTps:// DOI.ORG/10.1038/NRN1825

Lane, R. D., Reiman, E. M., Ahern, G. L., Schwartz, G. E. y Davidson, R. J. (1997). Neuroanatomica correlates of happiness, sadness, and disgust. The American Journal of Psychiatry, 154(7), 926-933. HTTPS://DOI.ORG/10.1176/AJP.154.7.926

Lang, A., Dhillon, K. y Dong, Q. (1995). The effects of emotional arousal and valence on television viewers' cognitive capacity and memory. Journal of Broadcasting \& Electronic Media, 39(3), 313-327. HTTPS://DOI.ORG/10.1080/08838159509364309

Lavan, N., Rankin, G., Lorking, N., Scott, S. y McGettigan, C. (2016). Neural correlates of the affective properties of spontaneous and volitional laughter types. Neuropsychologia, 95, 30-39. H T T P S : / / D O I. O R G / 10.1016 L.NEUROPSYCHOLOGIA.2016.12.012

Lewis, P. A., Critchley, H. D., Rotshtein, P. y Dolan, R. J. (2007). Neural correlates of processing valence and arousal in affective words. Cerebral Cortex, 17(3), 742-748. HTTPS://DOI.ORG/10.1093/CERCOR/BHKO24

Lisman, J. E. y Grace, A. A. (2005). The hippocampalVTA loop: Controlling the entry of information into long-term memory. Neuron, 46(5), 703-713. HTTPS://DOI.ORG/10.1016/J.NEURON.2005.05.002
Martin, R. A. (2007). The social psychology of humor. En The psychology of humor: An integrative approach (pp. 113-152). Elsevier. HTTPs:// DOI.ORG/10.1016/B978-012372564-6/50024-1

Martin, R. A. y Lefcourt, H. M. (1983). Sense of humor as a moderator of the relation between stressors and moods. Journal of Personality and Social Psychology, 45(6), 1313-1324.

Martin, R. A. y Lefcourt, H. M. (2004). Sense of humor and physical health: Theoretical issues, recent findings, and future directions. Humor, 17(1/2), $1-$ 20. HTTPS://PSYCNET.APA.ORG/DOI/10.1515/ HUMR.2004.005

Martin, R. A., Puhlik-Doris, P., Larsen, G., Gray, J. y Weir, K. (2003). Individual differences in uses of humor and their relation to psychological wellbeing: Development of the Humor Styles Questionnaire. Journal of Research in Personality, 37(1), 48-75. HTTPS://DOI.ORG/10.1016/Soog26566(02)00534-2

McGettigan, C., Walsh, E., Jessop, R., Agnew, Z. K. Sauter, D. A., Warren, J. E. y Scott, S. K. (2015). Individual differences in laughter perception reveal roles for mentalizing and sensorimotor systems in the evaluation of emotional authenticity. Cerebral Cortex, 25(1), 246-257. HTTPS://DOI.ORG/10.1093/CERCOR/BHT227

Meyer, M., Baumann, S., Wildgruber, D. y Alter, K. (2007). How the brain laughs. Behavioural Brain Research, 182(2), 245-260. HTTPS://DOI.ORG/ 10.1016/J.BBR.2007.04.023

Mickley, K. R. y Kensinger, E. A. (2008). Emotional valence influences the neural correlates associated with remembering and knowing. Cognitive, Affective, \& Behavioral Neuroscience, 8(2), 143-152. HTTPS://DOI.ORG/10.3758/CABN.8.2.143

Mobbs, D., Greicius, M. D., Abdel-Azim, E., Menon, V. y Reiss, A. L. (2003). Humor modulates the mesolimbic reward centers. Neuron, 40(5), 10411048. HTTPS://DOI.ORG/10.1016/So8966273(03)00751-7

Myers, D. G. y Diener, E. (1995). Who is happy? Psychological Science, 6(1), 10 19. HTTPs:// DOI.ORG/10.1111/J.1467-9280.1995.TB00298.X

Newcomer, J. W., Selke, G., Melson, A. K., Hershey, T., Craft, S., Richards, K. y Alderson, A. L. (1999). Decreased memory performance in healthy humans induced by stress-level cortisol treatment. Archives of General Psychiatry, 56(6), 527-533. HT TPS://PSYCNET.APA.ORG/DOI/10.1001/ ARCHPSYC. 56.6.527

Nothnagel, H. Z. (1889). Diagnose der Sehhügelerkrankungen. Z Klin Med, 16, 424-430. 
Oei, N. Y., Elzinga, B. M., Wolf, O. T., de Ruiter, M. B., Damoiseaux, J. S., Kuijer, J. P., Veltman, D. J., Scheltens, P. y Rombouts, S. A. (2007). Glucocorticoids decrease hippocampal and prefrontal activation during declarative memory retrieval in young men. Brain Imaging and Behavior, 1(1 2), 31-41.

Paré, D., Collins, D. R. y Pelletier, J. G. (2002). Amygdala oscillations and the consolidation of emotional memories. Trends in Cognitive Sciences, 6(7), 306-314. HTTPS://DOI.ORG/10.1016/S13646613(02)01924-1

Patil, A., Murty, V. P., Dunsmoor, J. E., Phelps, E. A. y Davachi, L. (2017). Reward retroactively enhances memory consolidation for related items. Learning \& Memory, 24(1), 65 69. HTTPS://DOI.ORG/ 10.1101/LM.042978.116

Phelps, E. A. (2004). Human emotion and memory: Interactions of the amygdala and hippocampal complex. Current Opinion in Neurobiology, 14(2), 198-202. HTTPS://DOI.ORG/10.1016/ L.CONB.2004.03.015

Phelps, E. A., Ling, S. y Carrasco, M. (2006). Emotion facilitates perception and potentiates the perceptual benefits of attention. Psychological Science, 17(4), 292-299. HTTPS://DOI.ORG/10.1111/ L.1467-9280.2006.01701.X

Pinheiro, A. P., Barros, C., Dias, M. y Kotz, S. A. (2017). Laughter catches attention! Biological Psychology, 130, 11-21. HTTPS://DOI.ORG/10.1016/ J.BIOPSYCHO.2017.09.012

Poeck, K. (1969). Pathophysiology of emotional disorders associated with brain damage. En P. Vinken y G. Bruyn (Eds.), Handbook of clinical neurology (pp. 3--343). North Holland.

Riem, M. M. E., van IJzendoorn, M. H., Tops, M., Boksem, M. A. S., Rombouts, S. A. R. B. y Bakermans-Kranenburg, M. J. (2012). No laughing matter: Intranasal oxytocin administration changes functional brain connectivity during exposure to infant laughter. Neuropsychopharmacology, 37(5), 1257-1266. HTTPS://DOI.ORG/10.1038/NPP.2011.313

Romundstad, S., Svebak, S., Holen, A. y Holmen, J (2016). A 15-year follow-up study of sense of humor and causes of mortality: The nord-trøndelag health study. Psychosomatic Medicine, 78(3), 345-353. HTTPS://DOI.ORG/10.1097/PSY.0000000000000275

Ruch, W. y Ekman, P. (2001). The expressive pattern of laughter. En A. Kaszniak (Ed.), Emotions, qualia, and consciousness (pp. 426-443). World Scientific.

Russell, J. A. (1980). A circumplex model of affect. Journal of Personality and Social Psychology, 39(6), 1161-1178.
Saarimäki, H., Gotsopoulos, A., Jääskeläinen, I. P., Lampinen, J., Vuilleumier, P., Hari, R., Sams, M. y Nummenmaa, L. (2016). Discrete neural signatures of basic emotions. Cerebral Cortex, 26(6), 2563-2573. HTTPS://DOI.ORG/10.1093/ CERCOR/BHVO 86

Saive, A. L., Royet, J. P., Ravel, N., Thévenet, M., Garcia, S. y Plailly, J. (2014). A unique memory process modulated by emotion underpins successful odor recognition and episodic retrieval in humans. Frontiers in Behavioral Neuroscience, 8 , 203. HTTPS://DOI.ORG/10.3389/FNBEH.2014.00203

Sander, K., Brechmann, A. y Scheich, H. (2003). Audition of laughing and crying leads to right amygdala activation in a low-noise fMRI setting. Brain Research Protocols, 11(2), 81-91. HTTPS:// DOI.ORG/10.1016/S1385-299X(03)00018-7

Sander, K. y Scheich, H. (2005). Left auditory cortex and amygdala, but right insula dominance for human laughing and crying. Journal of Cognitive Neuroscience, 17(10), 1519-1531. HTTPs:// DOI.ORG/10.1162/089892905774597227

Schmidt, S. R. (1994). Effects of humor on sentence memory. Journal of Experimental Psychology. Learning, Memory, and Cognition, 20(4), 953-967. HTTPS://DOI.ORG/10.1037/0278-7393.20.4.953

Schmidt, S. R. (2002). The humour effect: Differential processing and privileged retrieval. Memory, 10(2), 127-138. HTTPS://DOI.ORG/10.1080/ $\underline{09658210143000263}$

Schmidt, S. R. y Williams, A. R. (2001). Memory for humorous cartoons. Memory \& Cognition, 29(2), 305-311. HTTPS://DOI.ORG/10.3758/BF03194924

Scott, S. K., Lavan, N., Chen, S. y McGettigan, C. (2014). The social life of laughter. Trends in Cognitive Sciences, 18(12), 618-620. н нтTPs:// DOI.ORG/10.1016/I.TICS.2014.09.002

Sem-Jacobsen, C. W. (1968). Depth-electrographic stimulation of the human brain and behavior: From fourteen years of studies and treatment of Parkinson's Disease and mental disorders with implanted electrodes (No. 710). Thomas Springfield.

Shields, G. S., Sazma, M. A., McCullough, A. M. y Yonelinas, A. P. (2017). The effects of acute stress on episodic memory: A meta-analysis and integrative review. Psychological Bulletin, 143(6), 636-675. HTTPS://DOI.ORG/10.1037/BUL0000100

Small, D. M., Gregory, M. D., Mak, Y. E., Gitelman, D., Mesulam, M. M. y Parrish, T. (2003). Dissociation of neural representation of intensity and affective valuation in human gustation. Neuron, 39(4), 701-711. HTTPS://DOI.ORG/ $10.1016 /$ So896-6273(03)00467-7 
Sripada, C. S., Phan, K. L., Labuschagne, I., Welsh, R., Nathan, P. J. y Wood, A. G. (2013). Oxytocin enhances resting-state connectivity between amygdala and medial frontal cortex. The International Journal of Neuropsychopharmacology, 16(02), 255-260. HTTPS://DOI.ORG/10.1017/ $\underline{\mathrm{S} 1461145712000533}$

Strange, B. A. y Dolan, R. J. (2004). $\beta$-Adrenergic modulation of emotional memory-evoked human amygdala and hippocampal responses. Proceedings of the National Academy of Sciences, 101(31), 11454-11458. HTTPS://DOI.ORG/10.1073/ PNAS.0404282101

Suls, J. M. (1972). A two-stage model for the appreciation of jokes and cartoons: An information-processing analysis. En The psychology of humor (pp. 81-100). Elsevier. HTTPS:/ /DOI.ORG/10.1016/B978-0-12-288950-9.50010-9

Suzuki, H. y Heath, L. (2014). Impacts of humor and relevance on the remembering of lecture details. Humor, 27(1), 87-101. HTTPS://DOI.ORG/10.1515/ HUMOR-2013-0051

Szameitat, D. P., Darwin, C. J., Szameitat, A. J., Wildgruber, D. y Alter, K. (2011). Formant characteristics of human laughter. Journal of Voice, 25(1), 32-37. HTTPS://DOI.ORG/10.1016/ L.JVOICE.2009.06.010

Takahashi, M. y Inoue, T. (2009). The effects of humor on memory for non-sensical pictures. Acta Psychologica, 132(1), 80-84. HTTPS://DOI.ORG/ 10.1016/J.ACTPSY.2009.06.001

Tambini, A., Rimmele, U., Phelps, E. A. y Davachi, L. (2016). Emotional brain states carry over and enhance future memory formation. Nature Neuroscience, 20(2), 271-278. HTTPS://DOI.ORG/ $10.1038 / \mathrm{NN} .4468$

Tse, M. M. Y., Lo, A. P. K., Cheng, T. L. Y., Chan, E. K. K., Chan, A. H. Y. y Chung, H. S. W. (2010). Humor therapy: Relieving chronic pain and enhancing happiness for older adults. Journal of Aging Research, 2010, artículo 343574. HTTPS:// DOI.ORG/10.4061/2010/343574

Viinikainen, M., Jääskeläinen, I. P., Alexandrov, Y., Balk, M. H., Autti, T. y Sams, M. (2010). Nonlinear relationship between emotional valence and brain activity: Evidence of separate negative and positive valence dimensions. Human Brain Mapping, 31(7), 1030-1040. HTTPS://DOI.ORG/10.1002/HBM.20915
Vrticka, P., Black, J. M. y Reiss, A. L. (2013). The neural basis of humour processing. Nature Reviews Neuroscience, 14(12), 860-868. HTTPs://DOI.ORG/ 10.1038/NRN 3566

Wager, T. D., Phan, K. L., Liberzon, I. y Taylor, S. F. (2003). Valence, gender, and lateralization of functional brain anatomy in emotion: A metaanalysis of findings from neuroimaging. NeuroImage, 19(3), 513-531. HTTPs://DOI.ORG/ $\underline{10.1016 / \mathrm{S} 1053-8119(03) 00078-8}$

Warren, J. E., Sauter, D. A., Eisner, F., Wiland, J., Dresner, M. A., Wise, R. J. S., Rosen, S. y Scott, S. K. (2006). Positive emotions preferentially engage an auditory-motor " mirror " system. Journal of Neuroscience, 26(50), 13067-13075. HTTPs:// DOI.ORG/10.1523/JNEUROSCI.3907-06.2006

Watson, K. K., Matthews, B. J. y Allman, J. M. (2007). Brain activation during sight gags and languagedependent humor. Cerebral Cortex, 17(2), 314-324. HTTPS://DOI.ORG/10.1093/CERCOR/BHJ149

Wattendorf, E., Westermann, B., Lotze, M., Fiedler, K. y Celio, M. R. (2015). Insular cortex activity and the evocation of laughter. Journal of Comparative Neurology, 524(8), 1608-1615. HTTPS://DOI.ORG/ $10.1002 / \mathrm{CNE} .23884$

Wild, B., Rodden, F. A., Grodd, W. y Ruch, W. (2003). Neural correlates of laughter and humour. Brain, 126(10), 2121-2138.

Wyer, R. S. y Collins, J. E. (1992). A theory of humor elicitation. Psychological Review, 99(4), 663-688. HTTPS://DOI.ORG/10.1037/0033-295X.99.4.663

Yik, M. S. M., Russell, J. A. y Barrett, L. F. (1999). Structure of self-reported current affect: Integration and beyond. Journal of Personality and Social Psychology, 77(3), 600-619. HтTP:// DX.DOI.ORG/10.1037/0022-3514.77.3.600

Yue, X., Jiang, F., Lu, S. y Hiranandani, N. (2016). To be or not to be humorous? Cross cultural perspectives on humor. Frontiers in Psychology, 7, 1495. HTTPs:/ /DOI.ORG/10.3389/FPSYG.2016.01495

Zander-Schellenberg, T., Collins, I. M., Miché, M., Guttmann, C., Lieb, R. y Wahl, K. (2020). Does laughing have a stress-buffering effect in daily life? An intensive longitudinal study. PloS ONE, 15(7), e0235851. HTTPS://DOI.ORG/10.1371/ IOURNAL.PONE.0235851 